Original article

https://www.journal-imab-bg.org

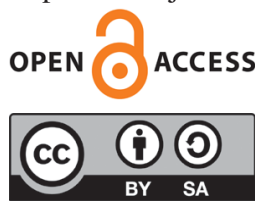

\title{
KINESITHERAPEUTIC APPROACH FOLLOWING SURGICAL TREATMENT IN CASES OF PARTIAL OR COMPLETE MUSCLES' RUPTURE OF THE ROTATOR CUFF
}

\author{
Gergana Nenova \\ Training sector of Kinesitherapy, Faculty of Public Health, Medical University \\ of Varna and Department of Orthopaedics and Traumatology, University \\ Hospital for Active Treatment "St. Marina" Varna, Bulgaria.
}

\begin{abstract}
:
Problems in the shoulder area are frequently encountered and result in significant limitations in the volume of movement, pain and inability to perform daily activities.

Material and methods: For a period of 18 months, 12 patients with rotator cuff tears have passed through the kinesitherapy office at the Department of Orthopaedics and Traumatology at St. Marina Marina University of Varna. Every one of these patients underwent surgical treatment and extensive kinesitherapy until about the 6th month after the operation. 7 of the patients are male, and 5 are female with the average age being 48 years for the men and 55.4 for the women.

Results: Satisfactory results were achieved in regards to the volume of joint movement in 10 of the patients as a result of the surgical treatment performed in the 12 cases (recovery of the rotator cuff with the help of shoulder fixation anchors attached to the affected tendons) and the extensive kinesitherapeutic treatment.

Conclusion: Applying the kinesitherapeutic treatment that is presented in the report, in the cases of patients with shoulder pathology, determines their complete functional recovery and improvement to the quality of life.
\end{abstract}

Keywords: rotator cuff, surgical treatment, kinesitherapy

\section{INTRODUCTION:}

The pathology observed in the shoulder complex determines the high percentage of soft tissue injuries affecting the tendon insertions of the rotator cuff's muscles supraspinatus, infraspinatus, teres minor, subscapularis [1]. These are the muscles involved in the inner rotation, the external rotation, and the lifting of the arm in abduction. They cover completely the head of the humerus completely, attaching themselves to the column humeri. The rupture of m.biceps brahii-caput longum is also very common. Treatment is determined depending on the location, extent, type, cause of the injury. and whether the limb will be used just for performing daily activities or in professional sports. Most often the supraspinatus suffers due to the fact of his anatomical position being between the shoulder bone and the acromion of the scapula, which is a predisposition for constant pressure. Thus the tendon becomes inflamed, starts to degenerate and can easily tear itself. In addition to a tear of the supraspinatus, other rotator cuff's muscles are susceptible to partial or complete tearings as a result of trauma or degenerative changes. The degree of the rupture and the type of treatment allow us to define the limitations in the volume of movement in the affected limb and to set the course of the kinesitherapeutic approach.

\section{MATERIAL AND METHODS}

Shoulder joint pathology is a common phenomenon in general practice, and in-depth knowledge of the problem would contribute to a correct and timely diagnosis. Creating an individual therapy plan is of significant importance for a proper recovery process and retrieving back working capacity. Such an approach includes a long-term strategy directed at the particular patient in which he participates and takes responsibility [2]. Studies in our clinical experience have shown that the age or genders are not relevant for the occurance of this pathology. Over the span of 18 months, we had the opportunity to observe patients affected with soft tissue injuries in the area of the shoulder joint who visited the Department of Orthopaedics and Traumatology at the University Hospital for Active Treatment "St. Marina". All patients have undergone surgical treatment and have been rehabilitated in the kinesitherapy office at the department. In this report, we only look at those who attended the kinesitherapy office at the department.

Over the course of a year and a half, the patients who visited the kinesitherapy office at the Orthopedics and Traumatology Clinic at St. Marina in Varna were 12, and all of them underwent surgical treatment and continuous 
kinesitherapy until about the 6th post-operative month. 7 of the patients are male, and 5 are female with the average age being 48 years for the men and 55.4 for the women. 11 of them had shoulder pathology as a result of an injury and 1 had endured a spontaneous rupture of rotator cuff's muscles as a result of degenerative changes in the tendons. The clinical cases of all patients show a front medial and / or deep shoulder pain, which intensifies at night and disturbs patients during sleeping. The ache remains in the daytime as well, causing discomfort as the patients are unable to perform some movements and sometimes radiates on the front surface of the proximal humerus. Some patients report "snapping" and "popping" in the joint and despite the use of conservative treatment, this symptom, along with the ongoing pain, areaffected only short-term. The available tests from the practice are characterized by high sensitivity, and low specificity and X-rays do not inform about possible ruptures of the rotator cuff. NMR and MRI are capable of providing more accurate information, but the most precise method for diagnosis and subsequent treatment is shoulder arthroscopy [3, 4, 5].

A partial or total rupture of the supraspinatus was observed in 8 of the 12 cases, 4 patients have tears/ruptures of the infraspinatus, 6 instances of biceps brahii- caput longum rupture. There were 3 cases where the patients have endured just an isolated tear of the supraspinatus. In all other 9 cases that we were able to observe, 2 or more muscles were affected, and in 2 of the patients, due to a traumatic component, all the muscles of the rotator cuff were damaged. With the exception of one patient who entered the clinic in an emergency for operative treatment due to a combined trauma, the remaining 11 had had long-term damage in the shoulder area. All of them had undergone conservative treatment, including both medication and physiotherapeutic treatment that had resulted in short pain relief and unsatisfactory movement capacity. The outcome of the surgical treatment (recovery of the rotator cuff with the help of shoulder fixation anchors attached to the affected tendons) was positive, and so was the post-operative therapeutic treatment in regards the volume of movement in the joint. Only in one of the cases the operative intervention was performed as tenodesis of the biceps brahii 's tendon and debridement and not as reinsertion of the intraspinal lesion of supraspinatus because the patient was a professional volleyball player.

The kinesitherapeutic treatment starts on the day after the surgery. It includes active kinesitherapy of the intact distally located joints and carefully controlled passive kinesitherapy of the affected joint determined by the threshold of pain. Pendulum exercises are very important as they include initiating swinging from the torso in the glenohumeral joint to flexion and extension, abduction and adduction, rotations. We also apply suspension therapy in a spider cage as well as joint-mobilization techniques and isometry for activation of the abductors, adductors and rotators in the shoulder joint. The set of exercises and their load are in accordance with the surgical intervention. After the removal of surgical sutures, we add to the methods of the passive kinesitherapy a massage (prior to and after the procedures) as well as exercises from a position of lying on the back with devices for wrist and elbow. All patients during the first 4 to 6 weeks wear an orthosis that they take off three times a day in order to perform their set of exercises. In this period, it is important to be achieved passive movement capacity in the joint so that we can move on to the next stage of actively assisted and actively controlled exercises.

Fig. 1. 4th post-operative week.

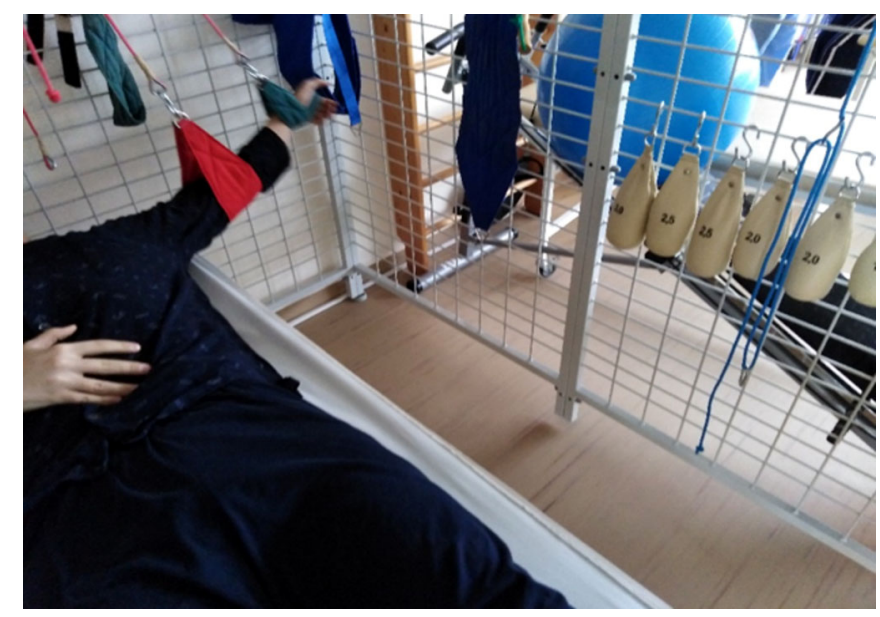

After the removal of the orthosis, which takes place at around the 6th-week post-surgery at the discretion of the orthopedist, the set of exercises is significantly expanded: active exercises with appliances and devices on all levels, Proprioceptive Neuromuscular Facilitation (PNF), elastic resistance exercises, etc. With the help of PNF, we aim to strengthen the central stimulation in volitional movements and to improve the activity of the rotator cuff's affected muscles [6]. When repeating volitional contraction facilitation of the hypotrophic muscle or muscle group, we reduce the synaptic resistance and stimulate the "voluntary" resistance pathways. In the subsequent recovery stages with the help of PNF, a direct volitional contraction of muscles is caused without additional stimuli to facilitate it as well as the application of manual-muscular techniques to prevent myogenic contractures [7,8]. Exercises with elastic resilience allow us to train all the joints of the upper limb because we do not rely on gravity but on the resistance of the strap [9]. This stimulates the elasticity of the soft tissues and the coordination of the limb during the exercises. With the help of elastic, bands we achieve influence upon muscle strength up to $30-35 \%$ at best in about $1-1,5$ months. 
Fig. 2. 6th post-operative week.

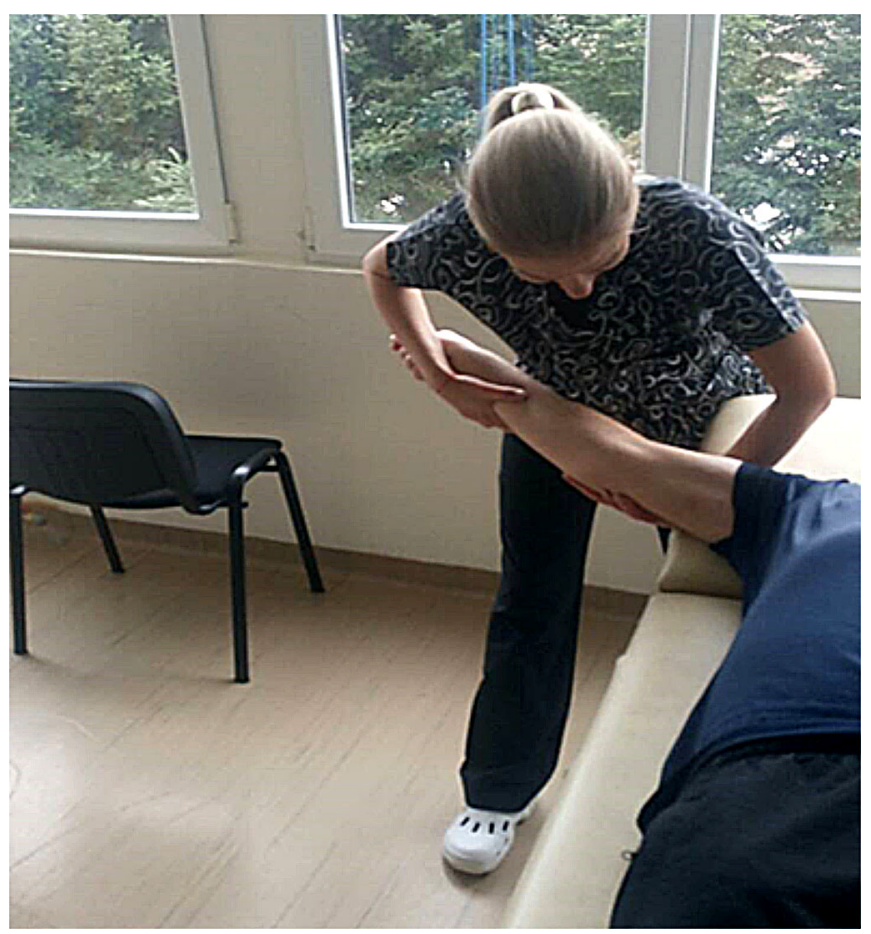

Fig. 3. 2nd post-operative month.

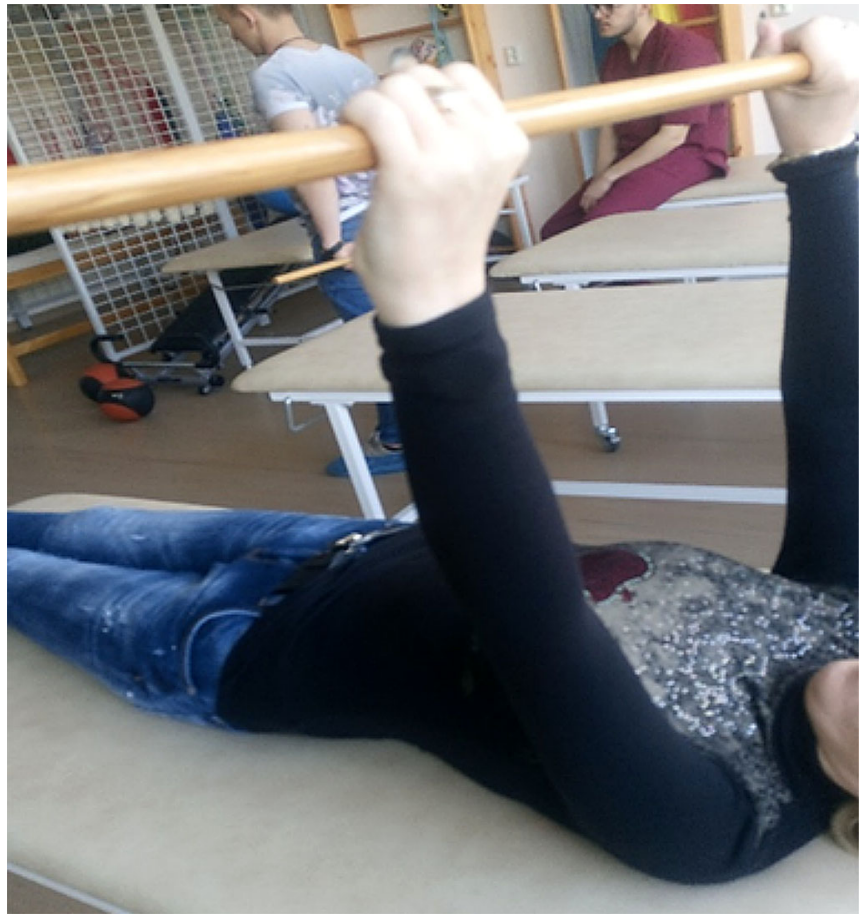

\section{DISCUSSION}

A properly prepared individual kinesitherapeutic set of exercises is essential for achieving positive results in the post-operative recovery period. Just resting and sparing the limb is, without a doubt, not beneficial to the patients at all. A number of authors recommend that treatment should engage the joint in exercising as early as possible to prevent subsequent complications $[10,11,12]$. The muscles forming the rotator cuff provide stability in the extremely incongruous glenohumeral joint. This is the reason why high-intensity loads are the cause of the suffering in this anatomical area. Well-selected kinesitherapeutic measurements and their purposeful application in accordance with the patient's individual characteristics as well as the properly assigned sequence of exercises during the various stages of the rehabilitation process are all key factors for recovering the function of the upper limb [13].

Fig. 4. 4th post-operative month.
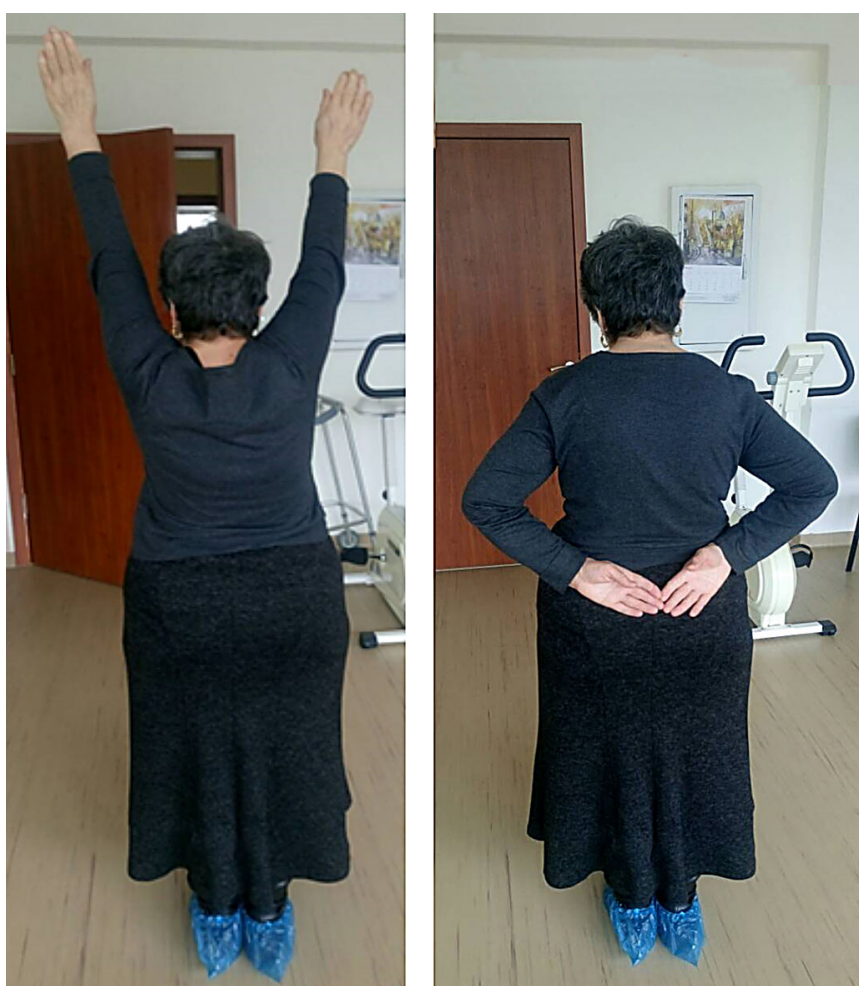

In the final stage of the treatment, after we have achieved the recovery of the active volume of movement in the joint, we direct the patients to sports activities like swimming and gym exercises and - in instances of professional athletes - a return to their respective sport. Two of the cases are professional volleyball players who were able to make a comeback to the volleyball court after the 6th month of their treatment. 8 of the patients recovered at a level that allowed them to perform their daily activities as well as working. However, in two of the 12 cases, we did not achieve the desired results due to the proximalisation of the humerus head. Thus the active volume of movement remained limited by the compensatory involvement of the scapula.

\section{CONCLUSION}

Shoulder pathology is one of the common problems in the orthopedic and kinesitherapeutic practice. The significant share of soft tissue damage in cases of partial 
tears or total rupture of the rotator cuff's tendon insertions is clearly a challenge for specialists. After the surgical treatment, it is important to apply a targeted individual kinesitherapeutic treatment for each stage of the recovery when striving to achieve maximum functional results. Applying the described sequence of kinesitherapeutic exercises determines the complete functional recovery and improvement of the quality of life of patients with shoulder pathology.

\section{REFERENCES:}

1. Deleva R. [Physical therapy in shoulder periarthropathies - impingement syndrome.] Monography. Mediatex-Pleven. 2018. p. 207. [in Bulgarian]

2. Mancheva P. [Back and shoulder pain - Therapeutic approaches in general practice.] General Medicine. 2004; 6(1):3-6. [in Bulgarian]

3. Dobrilov S, Zagorov M, Mihov K, Tabakov A, Nenova G. [Subopteral teodosis of the long head of $\mathrm{m}$ biceps brachii with bone anchor.] Ortopeadic and Traumatology. 2017; 54(2):328336. [in Bulgarian]

4. Murthi AM, Vosburgh CL, Neviaser TJ. The incidence of pathologic changes of the long head of the bceps tendon. J Shoulder Elbow Surg. 2000 Sep-Oct; 9(5):382-5. [PubMed] [Crossref]
5. Jordan RW, Saithna A. Physical examination tests and imaging studies based on arthroscopic assessment of the long head of biceps tendon are invalid. Knee Surg Sports Traumatol Arthrosc. 2017 Oct;25(10):3229-3236. [PubMed] [Crossref]

6. Bojinov S. [Neurology.] 2nd ed. Sofia; 1963 p. 19 - 20. [in Bulgarian]

7. Dimitrova E. [Use of manual muscular techniques in the treatment of myogenic contractions in the elbow joint.] Rehabilitation medicine and quality of life. 2007; 2: p.16-22. [in Bulgarian]

8. Mateev D. [Human physiology.] 2nd ed. Sofia: Medicine and Physical Education; 1962 p. 162. [in Bulgarian]

9. Cleeman E, Flatow EL. Shoulder dislocations in the young patient.
Orthop Clin North Am. 2000 Apr;31(2):217-29. [PubMed] [Crossref]

10. Ganchev M. [Clinical orthopedics.] Monography. K\&M - Sofia; 1997. [in Bulgarian]

11. Vladimirov B. [Orthopedics, traumatology and orthotics.] 1st ed. Medicine (Sofia). 2000, p.280-295. [in Bulgarian]

12. Halvadjian P. [Principles and Indications for the Use of Arthroscopy of the Shoulder Joint in Clinical Practice.] Orthopedics (Sofia) 2010. [in Bulgarian]

13. Popova D, Mitova St. Kinesitherapy to injuries of the rotator cuff. 11th International Scientific Conference. Veles. Activities in physical Education and sport. (Skopje, Macedonia.) 2014; 4(1):34-37.

Please cite this article as: Nenova G. Kinesitherapeutic approach following surgical treatment in cases of partial or complete muscles' rupture of the rotator cuff. J of IMAB. 2019 Jul-Sep;25(3):2628-2631.

DOI: https://doi.org/10.5272/jimab.2019253.2628

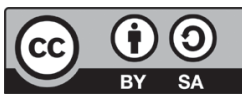

\author{
Address for correspondence: \\ Gergana Boncheva Nenova, \\ Training sector of Kinesitherapy, Faculty of public health, Medical University \\ of Varna and Department of Orthopaedics and Traumatology, University Hospital \\ for Active Treatment "St. Marina" Varna \\ 1, Hristo Smirnenski Str., 9002 Varna, Bulgaria. \\ E-mail: geri_nenova@yahoo.com
}

